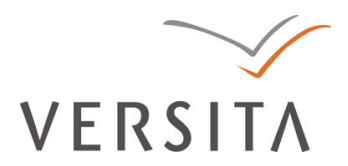

Folia Oeconomica Stetinensia

DOI: $10.2478 / \mathrm{v} 10031-012-0025-6$

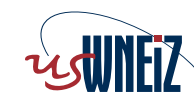

Wydzial Nauk Ekonomicznych i Zarządzania
Uniwersytetu Szczecińskiego

\title{
BETA COEFFICIENTS OF POLISH BLUE CHIP COMPANIES \\ IN THE PERIOD OF 2005-2011
}

\author{
Wiesław Dębski, Prof. \\ University of Finance and Management in Warsaw \\ Pawia 55, 01-030 Warszawa, Poland \\ e-mail:wdebski@vizja.pl
}

Ewa Feder-Sempach, Ph.D.

University of Lodz

Faculty of Economics and Sociology

POW 3/5, 90-255 Łódź, Poland

e-mail: ewafeder@wp.pl

Received 10 September 2012, Accepted 23 April 2013

\begin{abstract}
Risk plays a significant role in various aspects of financial decision throughout the world financial markets. Beta parameter is one of the commonly used coefficient to estimate the systematic risk associated with stocks. Beta is mostly calculated using single index market model by W. Sharpe.

This study examined the beta parameter under bull and bear market conditions on the Warsaw Stock Exchange (WSE). This paper analyses the beta responses for bad and good news for 44 stocks (14 stocks from the WIG20 index and 30 stocks from the mWIG40 index) over the last six years of trading at the WSE. Beta was calculated using monthly returns over the period 2005-2011, separately for the bull and the bear market. Our analysis finds strong evidence that beta is different in bull and bear market phase.
\end{abstract}

Keywords: beta coefficient, bull and bear market, Single-Index Market Model, Dual Beta Market Model.

JEL classification: G11, G01. 


\section{Introduction}

Beta coefficients are the most commonly used measures of systematic risk in connection with investments in shares. For many years indeed the analysts and practitioners of the financial markets put emphasis on different shaping of beta coefficients during the bull and bear market condition, depending on the main market trend. The division of the market into the bull market (growth) and the bear market (downturn) as a part of estimating parameters of the single-index Sharpe's model has been used since the 1970s not only in the American market.

The aim of the article is to point at differences in estimating beta parameters on the basis of the single-index model for the Polish blue chip listed companies in the periods of bull and bear market on the Warsaw Stock Exchange in 2005-2011. The empirical research covered in the article has been conducted on companies belonging to the indices WIG20 and mWIG40. The period of the research is specific because it embraces a strong upward trend in the initial years of the examined period in the Polish stock market and a period of the latest financial crisis during which the share prices fell significantly. The data for the research were obtained from Thomson Reuters database.

\section{Review of research conducted in the USA and on the emerging markets}

One of the first articles which discussed the distinction of beta coefficients due to the bull/bear market was an article by Robert Levy ${ }^{1}$. The research was conducted on the basis of 500 companies listed on the NYSE in the period of 1960-1970. The author analyzed a connection between the beta coefficient and the rate of return from the individual shares in the subsequent years of the sample. One of the conclusions that he presented was a suggestion to estimate beta coefficients for the bull market and the bear market separately, since the rates of return in each of those markets can be more precisely forecast with the use of the beta coefficient estimated for the bull market and the bear market respectively.

Another article in which the same division into the bull and bear market was used to estimate the beta coefficient was a paper by Frank Fabozzi and Jack Francis ${ }^{2}$. The main aim of the research conducted by them was to find an answer to the question whether the ratios were stable in the growth and decline period on the market. The analysis was made on the basis of the rates of return of 700 shares listed on the NYSE in the period of 1966-1970. The study showed the stability of beta coefficients and questioned the division into the bull and bear market.

Moon Kim and Kenton Zumwalt ${ }^{3}$ also dealt with volatility of the rate of return for the created portfolios and individual shares dividing the market into the growth market and the 
decline market. In their research the authors referred also to the results of Fabozzi and Francis, claiming that despite the fact that the beta coefficients on both markets did not differ in statistical sense, the changeability of rates of return in the bull and bear markets was different. They started with an assumption that investors strongly dislike risk and require a bonus for the risk in the bear market, whereas they themselves pay a bonus in the growth market. The results of the conducted research indicated that the beta coefficient estimated in the bear market is a better measure of the portfolio risk than the same ratio calculated for the entire sample period.

Among numerous research in the world referring to beta coefficient there are also the ones in which shaping of beta coefficient was analyzed in the periods of highs and falls in the emerging markets. For example Saumitra Bhaduri and Raja Durai ${ }^{4}$ examined the stability of the beta coefficient on the stock exchange in Bombay. To assess that parameter they used monthly rates of return of 78 shares listed on Bombay Stock Exchange in the period of 1999-2004. The result was convergent with the results of the research by Fabozzi and Francis from 1977 - the estimated values of beta parameter are not affected by the division of the market into the bull/bear market.

Another research into the emerging markets was conducted by Razvan Stefanescu, Costel Nistori Ramona Dumitriu ${ }^{5}$. They analyzed the estimates of beta parameter on the growth, decline and tranquil market for 10 shares listed on the stock exchange in Bucharest taking into account the daily rates of return in the period from $20^{\text {th }}$ January to $20^{\text {th }}$ July 2009 . The results indicated higher values of beta coefficients in the bull market and lower values in the bear market for the same shares. The average value of beta coefficients in the bull market was 1.135 , in the bear market 0.900 , and in the tranquil market 0.937 . The differences were explained by optimism of investors during bull market phase, which caused their willingness to take risk, and by pessimism occurring during lows ${ }^{6}$.

Similar research was carried out also on the Australian market for industrial companies and in other geographical regions in the world ${ }^{7}$. Numerous studies of beta coefficient have also been conducted in Poland and in other countries of the region ${ }^{8}$.

\section{Division of the market condition into the bear and the bull market}

The key problem for all the analyses of the beta coefficient on the growth and decline markets is a division of the examined period into the bull market and the bear market. There are a few methods of such classification, but most commonly it is made on the basis of the values 
of the rate of return or a moving average rate of return for an examined period. The divisions are as follows:

1. SD (Simple Definition of the Markets). The simplest method of the division assumes that if the rate of return from the index which illustrates the market is higher in the period $(t)$ than in the period $(t-1)$, then the period $(\mathrm{t})$ is a bull market and the period $(t-1)$ is a bear market.

2. UD (Up and Down Markets). The UD method is as simple as the previous one since it assumes that all the positive rates of return achieved in the market index belong to the bull market, and the negative ones belong to the bear market.

3. SUD (Substantially Up and Down Months). The SUD method divides the examined period into three sub-periods. If the rate of return from the market index in the period $\mathrm{t}$ is higher than 0.5 of standard deviation of this rate in the period of the examined sample, then the period $t$ is classified as the bull market. If, however, the rate of return from index in period $t$ is lower than 0.5 of standard deviation of this rate in the period of the examined sample, then such a period is classified as a bear market. The periods where the rates of return from the market index do not fluctuate either up or down by more than 0.5 of standard deviation are not taken into account.

4. PT (Peak and Trough Method). The PT method is based on the use of a moving average rate of return from the market index in the period $t\left(r_{m t}\right)$. In this method there are two identified moments, that is peak and trough. The peak in the period $(t)$ is defined if:

$$
\left\{r_{m, t-2}, r_{m, t-1}\right\}<r_{m t}>\left\{r_{m, t+1}, r_{m, t+2}\right\}
$$

and the trough in the period $(\mathrm{t})$ if:

$$
\left\{r_{m, t-2}, r_{m, t-1}\right\}>r_{m t}<\left\{r_{m, t+1}, r_{m, t+2}\right\}
$$

Having defined the peak and trough, the period between the trough and peak is classified as the bull market, and the period between the peak and the trough as the bear market 9 .

5. An intersection of the moving average with the stock exchange index. The bull market in this method is defined as a period in which the stock exchange index is above 250 -day ${ }^{10}$ moving average, and the bear market if it is lower than that. According to this classification, an investor occupying a long position in the bull market gains profits, and an investor who holds a short position in the bear market gains profits. This method has advantages, because it is easy to apply and quickly indicates a change of the market trend ${ }^{11}$. 


\section{Methodology}

The research involved selected stocks of the companies listed on the Warsaw Stock Exchange in the period of 2005-2011, belonging to indexes gathering the largest companies - the WIG20 (14 companies) and the mWIG40 (30 companies). The selected companies had Stock Exchange quotations throughout the whole examined period. Monthly rates of return were assumed, these were calculated on the basis of the closing rates from the last day of each month according to the following formula, not considering the return from dividend, that is:

$$
R_{i t}=\left(\ln P_{i, t}-\ln P_{i, t-1}\right) \cdot 100
$$

where:

$R_{i t} \quad-$ monthly rate of return of the $i$-th security in the period $t$,

$P_{i t} \quad-$ price of the $i$-th security in the period $t$,

$P_{i(t-1)}-$ price of the $i$-th security in the period $t-1$.

The beta coefficients were estimated on the basis of a Single-Index Market Model (SIMM) by Sharpe ${ }^{12}$ :

$$
R_{i t}=\alpha_{i}+\beta_{i} R_{W I G t}+\varepsilon_{i t}
$$

where:

$R_{W I G t}-$ return rate from the Stock Exchange index WIG in month $t$,

$\alpha_{i} \quad-$ alfa coefficient,

$\beta_{i}-$ beta coefficient,

$e_{i t} \quad-$ random term in month $t$.

The equation SIMM has a linear form and it describes the relation between the rate of return from an individual share, and the rate of return of the market portfolio, which is the WIG index representing the entire stock market in Poland. The rates of return of companies from the WIG20 and the mWIG40 were used to estimate the parameters of the above equation which was assessed with an Ordinary Least Squares method (OLS).

In turn, the market was divided into two periods - the bull market (growth) and the bear market (decline). The division was made according to the method based on the intersection points of the moving average ${ }^{13}$ with the stock exchange index. Those points were determined on the basis of an intersection of the 14-day moving average with the WIG index, which is illustrated in Figure 1. 


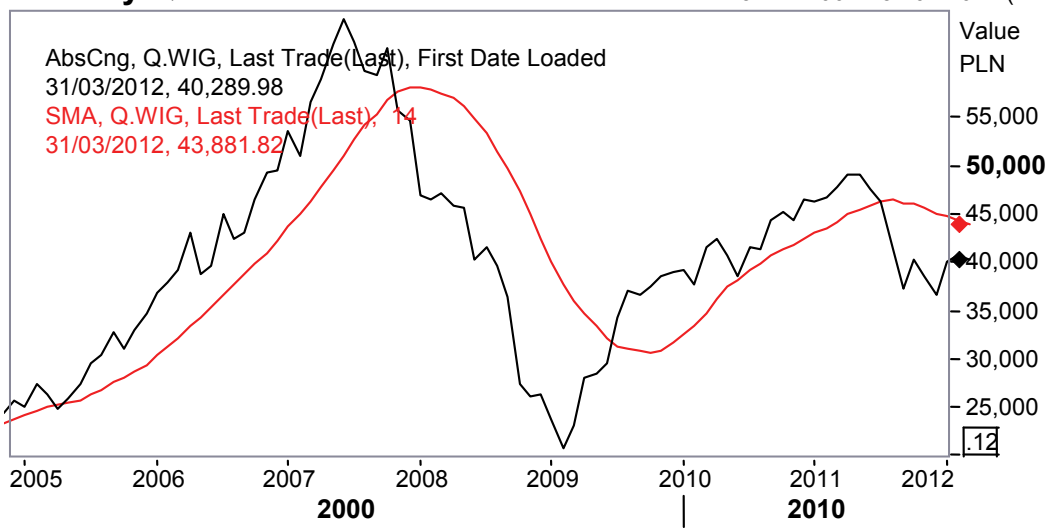

Fig. 1. Index values of WIG and 14-day moving average in the period 2005-2011

Source: own calculations on the basis of data from Thomson Reuters.

A period qualified as a bull market is:

1.01.2005 - 30.11.2007 and 31.07.2009 - 31.07.2011, while a period qualified as a bear market is:

31.12.2007 - 30.06.2009 and 31.08.2011 - 31.01.2012.

In order to estimate beta parameters in the bull and bear market, DBMM (Dual Beta Market Model $)^{14}$ was used, such as:

$$
R_{i t}=\alpha_{i}+\beta_{i 1} D_{1} R_{W I G t}+\beta_{i 2} D_{2} R_{W I G t}+\varepsilon_{i t}
$$

where:

$D_{1}=1$ for the bull market and 0 for the bear market,

$D_{2}=0$ for the bull market and 1 for the bear market,

$\beta_{i 1}$ - beta coefficient for the bull market,

$\beta_{i 2}-$ beta coefficient for the bear market.

The parameters of the DBMM equation were also determined with the OLS method. The estimation results of both the above models are presented in Tables 1 and 2 as divided into industries according to the classification at the Warsaw Stock Exchange. 
Table 1. Estimates of beta coefficients of the WIG20 index companies from different sectors (model 1 and 2) in the periods of the bull market and the bear market in 2005-2011

\begin{tabular}{|c|c|c|c|c|c|c|}
\hline Company & $\begin{array}{l}\text { Beta - bull } \\
\text { market } \\
(t)\end{array}$ & $\begin{array}{c}\text { Beta - bear } \\
\text { market } \\
(t)\end{array}$ & $\begin{array}{l}R^{2} \\
(F)\end{array}$ & $\begin{array}{c}\text { Beta - whole } \\
\text { period } \\
(t)\end{array}$ & $\begin{array}{l}R^{2} \\
(F)\end{array}$ & Sector \\
\hline ASSECOPOL & $\begin{array}{c}0.90 \\
(5.70) \\
\end{array}$ & $\begin{array}{c}0.76 \\
(5.51) \\
\end{array}$ & $\begin{array}{c}0.45 \\
(33.3) \\
\end{array}$ & $\begin{array}{c}0.82 \\
(8.17) \\
\end{array}$ & $\begin{array}{c}0.45 \\
(66.7) \\
\end{array}$ & IT \\
\hline BRE & $\begin{array}{l}-0.19 \\
(0.58)\end{array}$ & $\begin{array}{c}0.40 \\
(1.41)\end{array}$ & $\begin{array}{c}0.02 \\
(1.13)\end{array}$ & $\begin{array}{c}0.15 \\
(0.69)\end{array}$ & $\begin{array}{c}0.01 \\
(0.48)\end{array}$ & banks \\
\hline GETIN & $\begin{array}{c}1.00 \\
(4.86)\end{array}$ & $\begin{array}{c}1.74 \\
(9.71)\end{array}$ & $\begin{array}{c}0.60 \\
(61.8) \\
\end{array}$ & $\begin{array}{c}1.42 \\
(10.43)\end{array}$ & $\begin{array}{c}0.57 \\
(108) \\
\end{array}$ & banks \\
\hline HANDLOWY & $\begin{array}{c}0.67 \\
(3.60)\end{array}$ & $\begin{array}{c}1.17 \\
(7.20)\end{array}$ & $\begin{array}{c}0.46 \\
(33.9)\end{array}$ & $\begin{array}{c}0.95 \\
(7.87)\end{array}$ & $\begin{array}{c}0.43 \\
(62.0)\end{array}$ & banks \\
\hline PEKAO & $\begin{array}{c}1.07 \\
(8.11)\end{array}$ & $\begin{array}{c}1.34 \\
(11.72)\end{array}$ & $\begin{array}{c}0.73 \\
(107) \\
\end{array}$ & $\begin{array}{c}1.22 \\
(14.45)\end{array}$ & $\begin{array}{c}0.72 \\
(208)\end{array}$ & banks \\
\hline PKOBP & $\begin{array}{c}1.13 \\
(8.85)\end{array}$ & $\begin{array}{c}1.31 \\
(11.8)\end{array}$ & $\begin{array}{c}0.74 \\
(115)\end{array}$ & $\begin{array}{c}1.23 \\
(15.1)\end{array}$ & $\begin{array}{c}0.74 \\
(229)\end{array}$ & banks \\
\hline GTC & $\begin{array}{c}0.99 \\
(5.03)\end{array}$ & $\begin{array}{c}1.16 \\
(6.75)\end{array}$ & $\begin{array}{c}0.48 \\
(37.4)\end{array}$ & $\begin{array}{c}1.09 \\
(8.67)\end{array}$ & $\begin{array}{c}0.48 \\
(75.0)\end{array}$ & developers \\
\hline KGHM & $\begin{array}{c}1.21 \\
(5.02)\end{array}$ & $\begin{array}{c}1.49 \\
(7.11)\end{array}$ & $\begin{array}{c}0.50 \\
(40.1) \\
\end{array}$ & $\begin{array}{c}1.37 \\
(8.93)\end{array}$ & $\begin{array}{c}0.49 \\
(79.7) \\
\end{array}$ & raw materials industry \\
\hline PKNORLEN & $\begin{array}{c}1.02 \\
(7.25)\end{array}$ & $\begin{array}{c}0.92 \\
(7.49)\end{array}$ & $\begin{array}{c}0.59 \\
(57.5) \\
\end{array}$ & $\begin{array}{c}0.97 \\
(10.8)\end{array}$ & $\begin{array}{c}0.59 \\
(115)\end{array}$ & fuel industry \\
\hline LOTOS & $\begin{array}{c}0.93 \\
(3.47)\end{array}$ & $\begin{array}{c}1.20 \\
(5.19)\end{array}$ & $\begin{array}{c}0.34 \\
(20.5) \\
\end{array}$ & $\begin{array}{c}1.08 \\
(6.39)\end{array}$ & $\begin{array}{c}0.33 \\
(40.7) \\
\end{array}$ & fuel industry \\
\hline PGNIG & $\begin{array}{c}0.43 \\
(2.82)\end{array}$ & $\begin{array}{c}0.39 \\
(2.91)\end{array}$ & $\begin{array}{c}0.18 \\
(8.71) \\
\end{array}$ & $\begin{array}{c}0.41 \\
(4.19)\end{array}$ & $\begin{array}{c}0.18 \\
(17.5) \\
\end{array}$ & fuel industry \\
\hline PBG & $\begin{array}{c}0.90 \\
(3.30)\end{array}$ & $\begin{array}{c}1.00 \\
(4.24)\end{array}$ & $\begin{array}{c}0.27 \\
(15.2) \\
\end{array}$ & $\begin{array}{c}0.96 \\
(5.55)\end{array}$ & $\begin{array}{c}0.27 \\
(30.7) \\
\end{array}$ & construction industry \\
\hline TPSA & $\begin{array}{c}0.83 \\
(6.13)\end{array}$ & $\begin{array}{c}0.08 \\
(0.71)\end{array}$ & $\begin{array}{c}0.32 \\
(19.3)\end{array}$ & $\begin{array}{c}0.41 \\
(4.33)\end{array}$ & $\begin{array}{c}0.19 \\
(18.7)\end{array}$ & telecommunications \\
\hline TVN & $\begin{array}{c}0.98 \\
(5.10)\end{array}$ & $\begin{array}{c}0.79 \\
(4.71) \\
\end{array}$ & $\begin{array}{c}0.39 \\
(25.5) \\
\end{array}$ & $\begin{array}{c}0.87 \\
(7.13)\end{array}$ & $\begin{array}{c}0.38 \\
(50.7) \\
\end{array}$ & media \\
\hline
\end{tabular}

$t$ - estimate of t-statistics, $F$ - estimate of $F$-statistics, $R^{2}$ - coefficient of determination.

Source: own calculations.

Table 2. Estimates of beta coefficients of the mWIG40 index companies from different sectors (model 1 and 2) in the periods of the bull market and the bear market in 2005-2011

\begin{tabular}{|c|c|c|c|c|c|l|}
\hline Company & $\begin{array}{c}\text { Beta }- \text { bull } \\
\text { market } \\
(t)\end{array}$ & $\begin{array}{c}\text { Beta- bear } \\
\text { market } \\
(t)\end{array}$ & $(F)$ & $\begin{array}{c}R^{2} \\
\text { period } \\
(\mathrm{t})\end{array}$ & $\begin{array}{c}\text { Beta }- \text { whole } \\
(F)\end{array}$ & Sector \\
\hline AGORA & 2 & 3 & 4 & 5 & 6 & 7 \\
\hline KREDYT B & 0.88 & 1.14 & 0.41 & 1.02 & 0.41 & media \\
& $(4.08)$ & $(6.10)$ & $(28.3)$ & $(7.49)$ & $(56.0)$ & \\
\hline INGBSK & 1.07 & 1.29 & 0.44 & 1.20 & 0.44 & banks \\
& $(4.50)$ & $(6.29)$ & $(31.6)$ & $(7.95)$ & $(63.1)$ & \\
\hline MILLENNIUM & 1.02 & 1.25 & 0.55 & 1.15 & 0.54 & banks \\
& $(5.55)$ & $(7.82)$ & $(48.6)$ & $(9.82)$ & $(96.5)$ & \\
& 1.46 & 1.99 & 0.72 & 1.75 & 0.70 & banks \\
& $(7.49)$ & $(11.69)$ & $(101)$ & $(13.9)$ & $(192)$ & \\
\hline
\end{tabular}




\begin{tabular}{|c|c|c|c|c|c|c|}
\hline 1 & 2 & 3 & 4 & 5 & 6 & 7 \\
\hline BUDIMEX & $\begin{array}{c}0.84 \\
(3.55)\end{array}$ & $\begin{array}{c}0.49 \\
(2.35)\end{array}$ & $\begin{array}{c}0.19 \\
(9.59)\end{array}$ & $\begin{array}{c}0.64 \\
(4.23)\end{array}$ & $\begin{array}{c}0.18 \\
(17.9)\end{array}$ & construction \\
\hline POLIMEXMS & $\begin{array}{c}0.90 \\
(3.82)\end{array}$ & $\begin{array}{c}1.49 \\
(7.29)\end{array}$ & $\begin{array}{c}0.47 \\
(35.5)\end{array}$ & $\begin{array}{c}1.23 \\
(8.11)\end{array}$ & $\begin{array}{c}0.44 \\
(65.7)\end{array}$ & construction \\
\hline BIOTON & $\begin{array}{c}0.91 \\
(2.34)\end{array}$ & $\begin{array}{c}0.85 \\
(2.52)\end{array}$ & $\begin{array}{c}0.13 \\
(6.27)\end{array}$ & $\begin{array}{c}0.88 \\
(3.56)\end{array}$ & $\begin{array}{c}0.13 \\
(12.6)\end{array}$ & $\begin{array}{l}\text { pharmaceutical } \\
\text { industry }\end{array}$ \\
\hline $\mathrm{CIECH}$ & $\begin{array}{c}1.28 \\
(4.52) \\
\end{array}$ & $\begin{array}{c}1.32 \\
(5.38)\end{array}$ & $\begin{array}{c}0.39 \\
(26.1)\end{array}$ & $\begin{array}{c}1.30 \\
(7.27)\end{array}$ & $\begin{array}{c}0.39 \\
(52.8)\end{array}$ & chemical industry \\
\hline SYNTHOS & $\begin{array}{c}0.86 \\
(2.95)\end{array}$ & $\begin{array}{c}1.08 \\
(4.28)\end{array}$ & $\begin{array}{c}0.26 \\
(14.2)\end{array}$ & $\begin{array}{c}0.98 \\
(5.33)\end{array}$ & $\begin{array}{c}0.26 \\
(28.4)\end{array}$ & chemical industry \\
\hline PULAWY & $\begin{array}{c}0.84 \\
(3.43) \\
\end{array}$ & $\begin{array}{c}0.96 \\
(4.52) \\
\end{array}$ & $\begin{array}{c}0.30 \\
(17.0) \\
\end{array}$ & $\begin{array}{c}0.91 \\
(5.86) \\
\end{array}$ & $\begin{array}{c}0.29 \\
(34.2) \\
\end{array}$ & chemical industry \\
\hline SWIECIE & $\begin{array}{c}0.72 \\
(3.18)\end{array}$ & $\begin{array}{c}0.43 \\
(2.23)\end{array}$ & $\begin{array}{c}0.16 \\
(7.97)\end{array}$ & $\begin{array}{c}0.55 \\
(3.89)\end{array}$ & $\begin{array}{c}0.16 \\
(15.1)\end{array}$ & wood industry \\
\hline EMPERIA & $\begin{array}{c}0.32 \\
(1.15)\end{array}$ & $\begin{array}{c}0.90 \\
(3.67)\end{array}$ & $\begin{array}{c}0.16 \\
(7.65)\end{array}$ & $\begin{array}{c}0.65 \\
(3.59)\end{array}$ & $\begin{array}{c}0.14 \\
(12.8)\end{array}$ & retail trade \\
\hline $\mathrm{CCC}$ & $\begin{array}{c}1.00 \\
(4.68)\end{array}$ & $\begin{array}{c}0.38 \\
(2.03)\end{array}$ & $\begin{array}{c}0.25 \\
(13.5)\end{array}$ & $\begin{array}{c}0.65 \\
(4.65)\end{array}$ & $\begin{array}{c}0.21 \\
(21.6)\end{array}$ & retail trade \\
\hline EUROCASH & $\begin{array}{c}0.60 \\
(2.93) \\
\end{array}$ & $\begin{array}{c}0.65 \\
(3.68) \\
\end{array}$ & $\begin{array}{c}0.22 \\
(11.7) \\
\end{array}$ & $\begin{array}{c}0.63 \\
(4.86) \\
\end{array}$ & $\begin{array}{c}0.22 \\
(23.6) \\
\end{array}$ & retail trade \\
\hline LPP & $\begin{array}{c}1.05 \\
(4.10)\end{array}$ & $\begin{array}{c}0.90 \\
(4.04)\end{array}$ & $\begin{array}{c}0.30 \\
(17.5)\end{array}$ & $\begin{array}{c}0.96 \\
(5.94)\end{array}$ & $\begin{array}{c}0.30 \\
(35.2)\end{array}$ & retail trade \\
\hline $\mathrm{ECHO}$ & $\begin{array}{c}1.56 \\
(7.05) \\
\end{array}$ & $\begin{array}{c}1.32 \\
(6.85) \\
\end{array}$ & $\begin{array}{c}0.56 \\
(51.2) \\
\end{array}$ & $\begin{array}{c}1.42 \\
(10.11)\end{array}$ & $\begin{array}{c}0.55 \\
(102) \\
\end{array}$ & developers \\
\hline IMPEXMET & $\begin{array}{c}1.38 \\
(4.98)\end{array}$ & $\begin{array}{c}1.52 \\
(6.29)\end{array}$ & $\begin{array}{c}0.46 \\
(34.0)\end{array}$ & $\begin{array}{c}1.46 \\
(8.29) \\
\end{array}$ & $\begin{array}{c}0.46 \\
(68.6)\end{array}$ & metallurgical industry \\
\hline KETY & $\begin{array}{c}0.84 \\
(4.15)\end{array}$ & $\begin{array}{c}0.88 \\
(5.03)\end{array}$ & $\begin{array}{c}0.36 \\
(22.5)\end{array}$ & $\begin{array}{c}0.86 \\
(6.75)\end{array}$ & $\begin{array}{c}0.36 \\
(45.5)\end{array}$ & metallurgical industry \\
\hline BORYSZEW & $\begin{array}{c}1.62 \\
(4.40) \\
\end{array}$ & $\begin{array}{c}1.67 \\
(5.21) \\
\end{array}$ & $\begin{array}{c}0.38 \\
(24.6) \\
\end{array}$ & $\begin{array}{c}1.65 \\
(7.06) \\
\end{array}$ & $\begin{array}{c}0.38 \\
(49.8) \\
\end{array}$ & metallurgical industry \\
\hline MCI & $\begin{array}{c}1.42 \\
(4.66)\end{array}$ & $\begin{array}{c}1.96 \\
(7.40)\end{array}$ & $\begin{array}{c}0.50 \\
(40.2)\end{array}$ & $\begin{array}{c}1.73 \\
(8.84)\end{array}$ & $\begin{array}{c}0.49 \\
(78.2)\end{array}$ & other finances \\
\hline MIDAS & $\begin{array}{c}1.44 \\
(2.06)\end{array}$ & $\begin{array}{c}2.30 \\
(3.78)\end{array}$ & $\begin{array}{c}0.19 \\
(9.73)\end{array}$ & $\begin{array}{c}1.93 \\
(4.32) \\
\end{array}$ & $\begin{array}{c}0.19 \\
(18.7)\end{array}$ & other finances \\
\hline ORBIS & $\begin{array}{c}0.99 \\
(5.16)\end{array}$ & $\begin{array}{c}1.04 \\
(6.29)\end{array}$ & $\begin{array}{c}0.46 \\
(34.9)\end{array}$ & $\begin{array}{c}1.02 \\
(8.41)\end{array}$ & $\begin{array}{c}0.46 \\
(70.7)\end{array}$ & hotels and restaurants \\
\hline AMREST & $\begin{array}{c}1.03 \\
(4.70) \\
\end{array}$ & $\begin{array}{c}0.87 \\
(4.57)\end{array}$ & $\begin{array}{c}0.36 \\
(22.7)\end{array}$ & $\begin{array}{c}0.93 \\
(6.76)\end{array}$ & $\begin{array}{c}0.36 \\
(45.6)\end{array}$ & hotels and restaurants \\
\hline ROVESE & $\begin{array}{c}1.39 \\
(5.06)\end{array}$ & $\begin{array}{c}0.83 \\
(3.47)\end{array}$ & $\begin{array}{c}0.33 \\
(19.8)\end{array}$ & $\begin{array}{c}1.07 \\
(6.07)\end{array}$ & $\begin{array}{c}0.31 \\
(36.8)\end{array}$ & building materials \\
\hline CDRED & $\begin{array}{c}0.58 \\
(1.30)\end{array}$ & $\begin{array}{l}1.52 \\
(3.91)\end{array}$ & $\begin{array}{c}0.18 \\
(8.82)\end{array}$ & $\begin{array}{c}1.11 \\
(3.87)\end{array}$ & $\begin{array}{c}0.15 \\
(15.0)\end{array}$ & IT \\
\hline IDMSA & $\begin{array}{c}1.62 \\
(4.54)\end{array}$ & $\begin{array}{c}1.61 \\
(5.20)\end{array}$ & $\begin{array}{c}0.38 \\
(25.2)\end{array}$ & $\begin{array}{c}1.62 \\
(7.15)\end{array}$ & $\begin{array}{c}0.38 \\
(51.1)\end{array}$ & capital market \\
\hline INTERCARS & $\begin{array}{c}0.80 \\
(2.55) \\
\end{array}$ & $\begin{array}{c}1.70 \\
(6.24)\end{array}$ & $\begin{array}{c}0.37 \\
(23.7) \\
\end{array}$ & $\begin{array}{c}1.31 \\
(6.42) \\
\end{array}$ & $\begin{array}{c}0.33 \\
(41.1) \\
\end{array}$ & wholesale trade \\
\hline KOPEX & $\begin{array}{c}1.27 \\
(4.18)\end{array}$ & $\begin{array}{l}1.76 \\
(6.64)\end{array}$ & $\begin{array}{c}0.44 \\
(32.4)\end{array}$ & $\begin{array}{l}1.55 \\
(7.96)\end{array}$ & $\begin{array}{c}0.44 \\
(63.2)\end{array}$ & $\begin{array}{l}\text { electro-machinery } \\
\text { industry }\end{array}$ \\
\hline NETIA & $\begin{array}{c}0.42 \\
(2.29)\end{array}$ & $\begin{array}{c}0.58 \\
(3.64)\end{array}$ & $\begin{array}{c}0.19 \\
(9.72)\end{array}$ & $\begin{array}{c}0.51 \\
(4.38)\end{array}$ & $\begin{array}{c}0.19 \\
(19.1)\end{array}$ & telecommunications \\
\hline PEP & $\begin{array}{c}1.07 \\
(5.11)\end{array}$ & $\begin{array}{c}1.02 \\
(5.61)\end{array}$ & $\begin{array}{c}0.43 \\
(30.4)\end{array}$ & $\begin{array}{c}1.04 \\
(7.85)\end{array}$ & $\begin{array}{c}0.43 \\
(61.6)\end{array}$ & energy industry \\
\hline
\end{tabular}

$t$ - estimate of t-statistics, $F$ - estimate of $F$-statistics, $R^{2}$ - coefficient of determination.

Source: own calculations. 
Additionally, the decision was to check the evaluations of beta coefficients estimated on the basis of the model with dummy variables as follows:

$$
R_{i t}=\alpha_{i 1}+\alpha_{i 2} D_{1}+\beta_{i 1} R_{W I G t}+\beta_{i 2} D_{1} R_{W I G t}+\varepsilon_{i t}
$$

(the notations similar as before).

In this model, the variable $D_{1}$ takes value 1 for the periods of the bull market and value 0 otherwise. It means that the estimate of the parameter $\beta_{i 1}$ is an estimate of beta coefficient for the whole period of the sample and the estimate of $\beta_{i 2}$ signifies the size of change of beta coefficient in the period of the bull market in relation to the estimate of $\beta_{i 1}$. The results of the estimation of such a model are presented below in Tables 3 and 4 .

Table 3. Estimates of beta coefficients of the WIG20 index companies from different sectors (model 3) in the periods of the bull market and the bear market in 2005-2011

\begin{tabular}{|c|c|c|c|c|}
\hline Company & $\begin{array}{l}\text { Change of beta } \\
\text { for the bull market } \\
(t)\end{array}$ & $\begin{array}{c}\text { Beta } \\
\text { - whole period } \\
(t)\end{array}$ & $\begin{array}{l}R^{2} \\
(F)\end{array}$ & Sector \\
\hline ASSECOPOL & $\begin{array}{c}0.14 \\
(0.65)\end{array}$ & $\begin{array}{c}0.79 \\
(5.54)\end{array}$ & $\begin{array}{c}0.46 \\
(22.4)\end{array}$ & IT \\
\hline BRE & $\begin{array}{l}-0.55 \\
(-1.33)\end{array}$ & $\begin{array}{c}0.15 \\
(0.53)\end{array}$ & $\begin{array}{c}0.16 \\
(4.89)\end{array}$ & banks \\
\hline GETIN & $\begin{array}{l}-0.73 \\
(-2.6)\end{array}$ & $\begin{array}{c}1.68 \\
(9.06)\end{array}$ & $\begin{array}{c}0.61 \\
(42.0)\end{array}$ & banks \\
\hline HANDLOWY & $\begin{array}{c}-0.5 \\
(-1.93)\end{array}$ & $\begin{array}{l}1.16 \\
(6.87)\end{array}$ & $\begin{array}{c}0.46 \\
(22.4)\end{array}$ & banks \\
\hline PEKAO & $\begin{array}{l}-0.28 \\
(-1.57)\end{array}$ & $\begin{array}{c}1.38 \\
(11.7)\end{array}$ & $\begin{array}{c}0.73 \\
(72.7)\end{array}$ & banks \\
\hline PKOBP & $\begin{array}{l}-0.19 \\
(-1.07)\end{array}$ & $\begin{array}{c}1.34 \\
(11.6)\end{array}$ & $\begin{array}{c}0.74 \\
(76.8)\end{array}$ & banks \\
\hline GTC & $\begin{array}{l}-0.16 \\
(-0.6)\end{array}$ & $\begin{array}{l}1.13 \\
(6.3)\end{array}$ & $\begin{array}{l}0.48 \\
(25)\end{array}$ & developers \\
\hline KGHM & $\begin{array}{l}-0.28 \\
(-0.85)\end{array}$ & $\begin{array}{c}1.51 \\
(6.89) \\
\end{array}$ & $\begin{array}{c}0.50 \\
(26.5)\end{array}$ & raw materials industry \\
\hline PKNORLEN & $\begin{array}{c}0.11 \\
(0.55)\end{array}$ & $\begin{array}{c}0.91 \\
(7.12)\end{array}$ & $\begin{array}{c}0.59 \\
(37.9)\end{array}$ & fuel industry \\
\hline LOTOS & $\begin{array}{l}-0.27 \\
(-0.75)\end{array}$ & $\begin{array}{c}1.17 \\
(4.86)\end{array}$ & $\begin{array}{c}0.34 \\
(13.7)\end{array}$ & fuel industry \\
\hline PGNIG & $\begin{array}{c}0.05 \\
(0.22)\end{array}$ & $\begin{array}{c}0.38 \\
(2.74)\end{array}$ & $\begin{array}{c}0.18 \\
(5.75)\end{array}$ & fuel industry \\
\hline PBG & $\begin{array}{l}-0.1 \\
(-0.27)\end{array}$ & $\begin{array}{c}0.98 \\
(3.96)\end{array}$ & $\begin{array}{c}0.27 \\
(10.1)\end{array}$ & construction \\
\hline TPSA & $\begin{array}{c}0.75 \\
(4.02)\end{array}$ & $\begin{array}{l}0.08 \\
(0.7)\end{array}$ & $\begin{array}{c}0.32 \\
(12.7)\end{array}$ & telecommunications \\
\hline TVN & $\begin{array}{c}0.21 \\
(0.82)\end{array}$ & $\begin{array}{c}0.69 \\
(4.07)\end{array}$ & $\begin{array}{c}0.42 \\
(19.48)\end{array}$ & media \\
\hline
\end{tabular}

$t$ - estimate of t-statistics, $F$ - estimate of $F$-statistics, $R^{2}$ - coefficient of determination.

Source: own calculations. 
Table 4. Estimates of beta coefficients of the mWIG40 index companies from different sectors (model 3) in the periods of the bull market and the bear market in 2005-2011

\begin{tabular}{|c|c|c|c|c|}
\hline Company & $\begin{array}{c}\text { Change of beta for } \\
\text { the bull market } \\
(t)\end{array}$ & $\begin{array}{c}\text { Beta } \\
\text { - whole period } \\
(t)\end{array}$ & $\begin{array}{l}R^{2} \\
(F)\end{array}$ & Sector \\
\hline 1 & 2 & 3 & 4 & 5 \\
\hline AGORA & $\begin{array}{c}-0.26 \\
(-0.88)\end{array}$ & $\begin{array}{c}1.09 \\
(5.64)\end{array}$ & $\begin{array}{c}0.42 \\
(19.1)\end{array}$ & media \\
\hline KREDYT B & $\begin{array}{c}-0.22 \\
(-0.68)\end{array}$ & $\begin{array}{l}1.21 \\
(5.7)\end{array}$ & $\begin{array}{c}0.46 \\
(22.3)\end{array}$ & banks \\
\hline INGBSK & $\begin{array}{l}-0.23 \\
(-0.9)\end{array}$ & $\begin{array}{c}1.24 \\
(7.43)\end{array}$ & $\begin{array}{c}0.55 \\
(32.0)\end{array}$ & banks \\
\hline MILLENNIUM & $\begin{array}{c}-0.52 \\
(-1.94)\end{array}$ & $\begin{array}{c}1.94 \\
(11.0)\end{array}$ & $\begin{array}{c}0.72 \\
(67.6)\end{array}$ & banks \\
\hline BUDIMEX & $\begin{array}{c}0.35 \\
(1.08)\end{array}$ & $\begin{array}{c}0.54 \\
(2.51) \\
\end{array}$ & $\begin{array}{c}0.2 \\
(6.67)\end{array}$ & construction \\
\hline POLIMEXMS & $\begin{array}{c}-0.58 \\
(-1.82)\end{array}$ & $\begin{array}{l}1.42 \\
(6.7) \\
\end{array}$ & $\begin{array}{c}0.48 \\
(24.5) \\
\end{array}$ & construction \\
\hline BIOTON & $\begin{array}{c}0.07 \\
(0.13)\end{array}$ & $\begin{array}{c}0.78 \\
(2.23)\end{array}$ & $\begin{array}{c}0.14 \\
(4.33) \\
\end{array}$ & pharmaceutical industry \\
\hline $\mathrm{CIECH}$ & $\begin{array}{l}-0.04 \\
(-0.1)\end{array}$ & $\begin{array}{c}1.29 \\
(5.04)\end{array}$ & $\begin{array}{c}0.39 \\
(17.3)\end{array}$ & chemical industry \\
\hline SYNTHOS & $\begin{array}{l}-0.22 \\
(-0.55)\end{array}$ & $\begin{array}{c}1.04 \\
(3.96)\end{array}$ & $\begin{array}{c}0.26 \\
(9.55)\end{array}$ & chemical industry \\
\hline PULAWY & $\begin{array}{c}-0.13 \\
(-0.38)\end{array}$ & $\begin{array}{c}0.99 \\
(4.47)\end{array}$ & $\begin{array}{c}0.3 \\
(11.3)\end{array}$ & chemical industry \\
\hline SWIECIE & $\begin{array}{l}0.28 \\
(0.9)\end{array}$ & $\begin{array}{c}0.44 \\
(2.15)\end{array}$ & $\begin{array}{c}0.16 \\
(5.25)\end{array}$ & wood industry \\
\hline EMPERIA & $\begin{array}{l}-0.55 \\
(-1.47)\end{array}$ & $\begin{array}{c}0.8 \\
(3.17)\end{array}$ & $\begin{array}{c}0.18 \\
(5.91)\end{array}$ & retail trade \\
\hline $\mathrm{CCC}$ & $\begin{array}{c}0.63 \\
(2.17)\end{array}$ & $\begin{array}{c}0.32 \\
(1.66)\end{array}$ & $\begin{array}{l}0.26 \\
(9.5)\end{array}$ & retail trade \\
\hline EUROCASH & $\begin{array}{c}-0.05 \\
(-0.18)\end{array}$ & $\begin{array}{c}0.64 \\
(3.48) \\
\end{array}$ & $\begin{array}{c}0.22 \\
(7.71) \\
\end{array}$ & retail trade \\
\hline LPP & $\begin{array}{c}0.16 \\
(0.45)\end{array}$ & $\begin{array}{c}0.85 \\
(3.67)\end{array}$ & $\begin{array}{c}0.31 \\
(11.8) \\
\end{array}$ & retail trade \\
\hline $\mathrm{ECHO}$ & $\begin{array}{l}0.24 \\
(0.8)\end{array}$ & $\begin{array}{c}1.31 \\
(6.52)\end{array}$ & $\begin{array}{c}0.56 \\
(33.7) \\
\end{array}$ & developers \\
\hline IMPEXMET & $\begin{array}{c}-0.12 \\
(-0.33)\end{array}$ & $\begin{array}{c}1.44 \\
(5.75)\end{array}$ & $\begin{array}{c}0.47 \\
(23.4)\end{array}$ & metallurgical industry \\
\hline KETY & $\begin{array}{l}-0.05 \\
(-0.17) \\
\end{array}$ & $\begin{array}{c}0.89 \\
(4.86) \\
\end{array}$ & $\begin{array}{c}0.36 \\
(14.8) \\
\end{array}$ & metallurgical industry \\
\hline BORYSZEW & $\begin{array}{c}-0.04 \\
(-0.09)\end{array}$ & $\begin{array}{c}1.64 \\
(4.91)\end{array}$ & $\begin{array}{c}0.38 \\
(16.3) \\
\end{array}$ & metallurgical industry \\
\hline MCI & $\begin{array}{c}-0.52 \\
(-1.27)\end{array}$ & $\begin{array}{c}1.85 \\
(6.79)\end{array}$ & $\begin{array}{c}0.51 \\
(28.0)\end{array}$ & other finances \\
\hline MIDAS & $\begin{array}{c}-0.88 \\
(-0.92)\end{array}$ & $\begin{array}{c}2.44 \\
(3.86)\end{array}$ & $\begin{array}{c}0.2 \\
(6.69)\end{array}$ & other finances \\
\hline ORBIS & $\begin{array}{c}-0.07 \\
(-0.26)\end{array}$ & $\begin{array}{c}1.11 \\
(6.46)\end{array}$ & $\begin{array}{c}0.48 \\
(24.2)\end{array}$ & hotels and restaurants \\
\hline AMREST & $\begin{array}{c}0.17 \\
(0.57) \\
\end{array}$ & $\begin{array}{c}0.8 \\
(4.08) \\
\end{array}$ & $\begin{array}{c}0.37 \\
(15.8) \\
\end{array}$ & hotels and restaurants \\
\hline ROVESE & $\begin{array}{c}0.57 \\
(1.53)\end{array}$ & $\begin{array}{c}0.75 \\
(3.05) \\
\end{array}$ & $\begin{array}{c}0.34 \\
(13.7) \\
\end{array}$ & building materials \\
\hline
\end{tabular}




\begin{tabular}{|l|c|c|c|l|}
\hline \multicolumn{1}{|c|}{1} & 2 & 3 & 4 & \multicolumn{1}{|l|}{5} \\
\hline CDRED & -0.93 & 1.5 & 0.18 & IT \\
& $(-1.53)$ & $(3.71)$ & $(5.82)$ & \\
\hline IDMSA & 0.03 & 1.46 & 0.41 & capital market \\
& $(0.07)$ & $(4.59)$ & $(18.5)$ & \\
\hline INTERCARS & -0.9 & 1.73 & 0.37 & wholesale trade \\
& $(-2.12)$ & $(3.29)$ & $(15.7)$ & \\
\hline KOPEX & -0.48 & 1.74 & 0.45 & electro-machinery industry \\
& $(-1.16)$ & $(6.31)$ & $(21.4)$ & \\
\hline NETIA & -0.17 & 0.61 & 0.2 & telecommunications \\
& $(-0.66)$ & $(3.7)$ & $(6.64)$ & \\
\hline PEP & 0.05 & 1.03 & 0.43 & energy industry \\
& $(0.16)$ & $(5.45)$ & $(20.1)$ & \\
\hline
\end{tabular}

$t$ - estimate of t-statistics, $F$ - estimate of $F$-statistics, $R^{2}$ - coefficient of determination.

Source: own calculations.

\section{Conclusions}

In case of all the examined WIG20 index companies (Table 1) except one (BRE), the estimated beta coefficients are statistically significant, regardless of whether they were estimated for the whole sample period (model 1) or for the specified periods of the bull market or the bear market (model 2). This has been confirmed by the estimates of the statistics $t$, as well as by the statistics F. They adopt values confirming statistical significance on the level 0.99 . What must also be emphasized are relatively high values of the coefficient of determination $\mathrm{R}^{2}$ which in a definite majority of cases exceeds 0.40 . In case of 8 companies (except BRE) the beta coefficient adopts the value that is lower in the bull market than in the bear market. It signifies relatively low potential of growth in the time of market highs and relatively higher potential of decline in the time of market lows in relation to the WIG index in the examined period. In case of 5 analyzed companies this relation was reversed, whereas the biggest difference between the value of beta coefficient for the bull and bear market was 0.20 (TVN).

In case of 30 companies from the mWIG40 index being the subject of the analysis (Table 2 ) the estimated beta coefficients (model 1 and 2) are statistically significant (only in two cases the estimate of statistics $t$ is lower than 2), while the coefficients of determination for just 10 companies are higher than 0.40 . It signifies high stability of the estimated beta coefficients, also when divided into the bull and bear market. However the range of the description of the rate of return from a share of an examined company made by means of the return rate from the WIG index is considerably smaller than in the case of big companies (listed on the WIG20), which in turn may reflect a relatively weaker predictive power of the beta coefficient in the case of mWIG40-listed companies. In 20 companies the estimated beta coefficients have lower values 
in the bull market than in the bear market, and in 10 companies there is a reverse situation, which confirms the obtained relation for companies from the WIG20 index. In two cases the value of beta coefficient in the bull market was more than a half higher than in the bear market.

The results of the estimates of beta coefficients calculated on the basis of the model with changing beta parameter (model 3) for the bull market in fact confirm the evaluations obtained on the basis of models 1 and 2. The only characteristic aspect are relatively low estimates of the $t$-Student statistics standing at the parameter describing the change of beta coefficient for the bull market in relation to the whole period (parameter $\beta_{i 2}$ ), which would contradict the thesis about estimating beta coefficient for the bull and bear market on the Warsaw Stock Exchange. The emphasis should be put on the relatively high estimates of the $t$-Student statistics standing at the parameter $\beta_{i 1}$, as well as the $\mathrm{F}$ statistics and the fitness rate.

To sum up, the conducted research seems to be useful since it has shown a diversification of beta coefficients in the bull and bear markets for the blue chip companies in Poland in the period of 2005-2011. The estimated parameters should have a stronger predictive power in these markets than the parameters estimated for the whole market. Taking into account beta coefficients estimated for the whole period, in the group of the examined companies we can distinguish 24 aggressive and 20 defensive ones. The most aggressive companies involve the companies from the financial sector (GETIN, MIDAS, MCI), while the most defensive ones are telecommunication companies (TPSA, NETIA) and the PGNIG company operating in the fuel industry.

\section{Notes}

1 Levy (1974).

2 Fabozzi, Francis (1977).

3 Kim, Zumwalt (1979).

4 Bhaduri, Durai (2006).

5 Stefanescu, Nistor, Dumitriu (2009).

6 The presented results of the research from the Romanian market are not comparable with other ones due to the frequency of measurement and they are questionable due to the length of the sample period.

7 See Woodward, Anderson (2009).

8 See Tarczyński (2009); Witkowska (2008), pp. 143-154; Cwynar (2008).

9 Bhaduri, Durai (2006).

${ }^{10}$ In the American market the most commonly used is 250-day average, however in other research there are also used average values for shorter periods.

${ }^{11}$ Chong, Li, Chen, Hinich (2010).

12 Sharpe (1964). 
13 The division was made on the basis of a 14-day average, because an average from a longer period was a straight line and indicating the highs and lows was difficult.

${ }^{14}$ Sometimes the equation is defined as DBM. See Bhaduri, Durai (2006), p. 57.

\section{References}

Bhaduri, S. \& Durai. S. (2006). Asymmetric beta in bull and bear market conditions: evidence from India. Applied Financial Economics Letters, 2, 55-59.

Chong, T., Li, Z., Chen, H. \& Hinich M. (2010). An investigation of duration dependence in the American stock market cycle. Journal of Applied Statistics, 1408.

Cwynar, W. (2008). Personalizacja w pomiarze ryzyka rynkowego. e-Finanse, finansowy kwartalnik internetowy $\mathrm{nr} 3,1-10$.

Fabozzi, F. \& Francis, J. (1977). Stability Tests for Alphas and Betas over Bull and Bear Market Conditions. The Journal of Finance, 1093-1099.

Kim, M. \& Zumwalt, K. (1979). An Analysis of Risk In Bull and Bear Markets. Journal of Financial and Quantitative Analysis, 1015-1025.

Levy, R. (1974). Beta Coefficients as Predictors of Return. Financial Analysts Journal, 61-69.

Sharpe, W. (1964). Capital Asset Prices: a theory of market equilibrium under conditions of risk. Journal of Finance, Vol. XIX, 425-442.

Stefanescu, R., Nistor, C. \& Dumitriu, R. (2009). Asymmetric Responses of CAPM - Beta to the Bull and Bear Markets on the Bucharest Stock Exchange. Annals of the University of Petrosani, 257-262.

Tarczyński, W. (2009). O pewnym sposobie wyznaczania współczynnika beta na polskim rynku kapitałowym. Zeszyty Naukowe Uniwersytetu Szczecińskiego nr 561, Studia i Prace Wydziału Nauk Ekonomicznych i Zarządzania, 199-214.

Witkowska, D. (2008). Badanie stabilności współczynnika beta oszacowanego na podstawie prób o różnej długości. Rynek kapitałowy skuteczne inwestowanie. Studia i prace Wydziału Nauk Ekonomicznych i Zarządzania nr 9, Uniwersytet Szczeciński.

Woodward, G. \& Anderson, H. (2009). Does beta react to market conditions? Estimates of "bull" and "bear" betas using a nonlinear market model with an endogenous threshold parameter. Quantitative Finance, Vol. 9, No. 8, 913-924. 